\title{
Asociación entre la ingesta de nutrientes hematopoyéticos y el origen nutricional de la anemia en mujeres en edad fértil en Colombia
}

\author{
Luz Mariela Manjarrés, ${ }^{1}$ Abel Díaz ${ }^{2}$ y Alicia Carriquiry ${ }^{3}$
}

Forma de citar

Manjarrés LM, Díaz A, Carriquiry A. Asociación entre la ingesta de nutrientes hematopoyéticos y el origen nutricional de la anemia en mujeres en edad fértil en Colombia. Rev Panam Salud Publica. 2012;31(1):68-73.

RESUMEN Objetivo. Comparar el origen de la anemia nutricional según las variables sociodemográficas y analizar su asociación con la deficiencia en la ingesta de nutrientes hematopoyéticos. Métodos. Se utilizó la base de datos de la Encuesta Nacional de la Situación Nutricional de Colombia, 2005. Los datos se obtuvieron por muestreo complejo representativo de la población y se procesaron con el programa SPSS, v.15. Se seleccionaron mujeres en edad fértil con anemia y se clasificaron en dos grupos según la ferritina sérica. Se determinó la ingesta usual de nutrientes hematopoyéticos y el riesgo de deficiencia. Se compararon las proporciones de los tipos de anemia según las variables sociodemográficas utilizando la prueba F de Rao-Scott de segundo orden $(\mathrm{P}<0,05)$. Se analizó la asociación entre el origen de la anemia y la clasificación del nutriente mediante la razón de posibilidades (odds ratio, OR).

Resultados. Muestra: 595 mujeres. Predominó la anemia no ferropénica $(67,2 \%)$, sin diferencia estadística por variables sociodemográficas, excepto en la región Pacífica (anemia ferropénica, 52,1\%). La prevalencia de la deficiencia en la ingesta usual de nutrientes hematopoyéticos fue alta. No se encontró asociación significativa entre el déficit de consumo y el origen de la anemia.

Conclusiones. La anemia no ferropénica fue más frecuente, sin diferencia según los indicadores sociodemográficos excepto en la región Pacífica. Todas las mujeres presentaron alto riesgo de deficiencia en la ingesta usual de nutrientes hematopoyéticos, pero no se observó una asociación estadísticamente significativa entre la deficiencia y el origen de la anemia nutricional. Se justifica implementar programas orientados a mejorar el aporte de nutrientes y continuar la búsqueda de otras causas de la anemia nutricional diferentes a la deficiencia de hierro.

Palabras clave

Anemia; anemias nutricionales; ferritinas; avitaminosis; deficiencia de hierro; deficiencia de vitamina B; salud de la mujer; Colombia.

La anemia es una de las enfermedades más comunes y generalizadas en el mundo; este trastorno afecta a todos los

\footnotetext{
1 Universidad de Antioquia, Escuela de Nutrición y Dietética. Medellín, Colombia. La correspondencia se debe enviar a Manjarrés Luz Mariela, luzmanjarresc@gmail.com

2 Universidad de Antioquia. Medellín, Colombia.

3 Iowa State University, Statistics Department, Ames, Iowa. Estados Unidos de America.
}

grupos de población pero, especialmente, a las mujeres. Se estima que la prevalencia mundial de la anemia en las mujeres en edad fértil es de 30,2\%; en América Latina es de $23,5 \%$ (1) y en Colombia de $32,8 \%$ (2).

En las personas afectadas la anemia en general disminuye la capacidad física y de aprendizaje y la respuesta inmune (3, 4), pero sus secuelas son más negativas específicamente durante el embarazo. La anemia gestacional se ha asociado con aumento de la mortalidad materna, insuficiencia cardíaca, menor peso del niño al nacer, mayor riesgo de parto prematuro, menor tolerancia a las pérdidas de sangre durante el trabajo de parto y mayor tiempo de cicatrización de las heridas $(5,6)$. 
La mayoría de los países del mundo han considerado que la principal causa de la anemia es la deficiencia de hierro. Por esta razón, han llevado a cabo programas de fortificación de alimentos y suplementación con el fin de incrementar la ingesta de este nutriente y disminuir los altos índices de esta enfermedad. Aunque se han logrado avances, los esfuerzos realizados no han tenido el resultado esperado $(3,7)$. Debido a ello, los organismos internacionales recomiendan identificar otros orígenes de la anemia, tales como infecciones (en especial, paludismo), infestación por parásitos helmínticos y deficiencia en la ingesta de vitaminas y minerales hematopoyéticos $(6,8)$.

Dentro de los nutrientes diferentes al hierro que se han estudiado como factores que pueden contribuir con el desarrollo de anemia se destacan la vitamina A (9-11), el cobre $(9,12)$, la vitamina $B_{6}$, la vitamina $\mathrm{B}_{12}$, el ácido fólico (13-17), el cinc (18) y la vitamina $C$, que aumenta la absorción de hierro (19).

En consonancia con la propuesta de los organismos internacionales como la Organización Mundial de la Salud (OMS) y la Organización de las Naciones Unidas para la Alimentación y la Agricultura (FAO) (10), los objetivos de este estudio fueron comparar los tipos de anemia (ferropénica y no ferropénica) según las variables demográficas (edad, Sistema de Identificación de Potenciales Beneficiarios de Programas Sociales [SISBEN], área y región geográficas) y analizar la asociación entre el origen de la anemia nutricional y el riesgo de deficiencia en la ingesta de hierro, vitamina $A$, vitamina $B_{6}$, vitamina $B_{12}$, vitamina $C$, cobre y cinc en las mujeres en edad fértil en Colombia.

\section{MATERIALES Y MÉTODOS}

Se realizó un estudio de corte transversal analítico para explorar la asociación entre la ingesta de nutrientes y la anemia nutricional, basado en los resultados de la Encuesta Nacional de la Situación Nutricional de Colombia (ENSIN 2005) (20). A partir de una submuestra, de 15909 personas, incluidas para el componente de indicadores bioquímicos, se tomaron 595 mujeres de entre 14 y 49 años con diagnóstico de anemia.

Como variables sociodemográficas se consideraron dos grupos de edad (14 a 18 años y 19 a 49 años), tres grupos de SISBEN (el SISBEN, clasificado en seis niveles, se reagrupó en tres: SISBEN 1, SISBEN 2 y SISBEN 3 a 6), dos áreas (urbana y rural) y seis regiones geográficas (Atlántica, Oriental, Central, Pacífica, Bogotá y Orinoquia-Amazonia).

Los indicadores bioquímicos considerados fueron la hemoglobina $(\mathrm{Hb})$ y la ferritina. La medición de la hemoglobina se hizo en campo mediante el fotómetro portátil de operación manual HemoCue. Los valores se ajustaron según la altitud del lugar de residencia de las participantes, teniendo en cuenta la fórmula de Dirren. El criterio para definir anemia fue una $\mathrm{Hb}<12,0 \mathrm{~g} / \mathrm{dL}$ (2). Para determinar la ferritina se analizaron las muestras de sangre en un equipo ADVIA Centauro, que emplea el método de quimioluminiscencia natural. El criterio para definir la depleción de los depósitos de hierro fue una ferritina $<12,0 \mu \mathrm{g} / \mathrm{L}$. La proteína $C$ reactiva (PCR) fue normal en todas las mujeres participantes (2).

Las mujeres con $\mathrm{Hb}$ menor de $12,0 \mathrm{~g} /$ $\mathrm{dL}$ y ferritina inferior a $12,0 \mu \mathrm{g} / \mathrm{L}$ se clasificaron dentro del grupo de anemia ferropénica (15). Las que tuvieron $\mathrm{Hb}$ menor de $12,0 \mathrm{~g} / \mathrm{dL}$ y ferritina igual o superior a $12,0 \mu \mathrm{g} / \mathrm{L}$, en el de anemia no ferropénica.

\section{Ingesta de nutrientes hematopoyéticos}

Dietistas capacitadas en la técnica de recordatorio de 24 horas lo aplicaron a todas las mujeres y además realizaron un segundo recordatorio en otro día no consecutivo con el primero, a una submuestra conformada por el $9 \%$ de las participantes, seleccionada en forma aleatoria. El segundo recordatorio es necesario, junto con el primero, para poder estimar la variabilidad en el consumo de nutrientes tanto entre días como intrapersona, y poder así estimar el consumo habitual en la población. Para responder a la entrevistadora las participantes tuvieron que recordar todas las comidas, bebidas y suplementos que consumieron durante las 24 horas previas a la encuesta. Para precisar las cantidades ingeridas se emplearon modelos de alimentos, figuras geométricas y un álbum con fotografías de utensilios en tamaño real (21).

El aporte nutricional de cada alimento, bebida y suplemento se calculó utilizando el Programa de Evaluación de la Ingesta Dietética de la Escuela de Nutrición y Dietética de la Universidad de Antioquia (22). Para cada uno de los alimentos consumidos el programa calcula cuántas unidades de cada nutriente contienen las porciones ingeridas por el participante en las 24 horas previas a la entrevista.

El recordatorio, de 24 horas, capta el consumo de nutrientes de una persona durante un día, pero la ingesta diaria individual varía significativamente. Desde el punto de vista de la salud pública, lo que interesa es la ingesta promedio del nutriente por cada persona. Ese consumo promedio se denomina ingesta habitual o ingesta usual.

Las distribuciones de consumo habitual de hierro, vitamina $\mathrm{C}$ y cinc se estimaron con la metodología propuesta por Nusser y colaboradores (23). Se utilizó el programa PC-SIDE, versión 1.0 de 2004 (Departamento de Estadística de la Universidad del Estado de Iowa, Ames, Iowa, EUA). Este programa estima las distribuciones de consumos habituales en la población y el consumo habitual de un nutriente para cada individuo. El estimador del consumo habitual para cada individuo que produce el programa PC-SIDE tiene buenas propiedades estadísticas, y se denomina mejor predictor lineal insesgado (MPLI) del consumo habitual de una persona.

Debido a que los consumos de vitamina $A$, vitamina $B_{6} y$ vitamina $B_{12}$, en esta muestra, se caracterizaron por su alta variabilidad y asimetría, fue necesario emplear otra metodología estadística para estimar las distribuciones de consumo habitual grupal y el de cada persona. Para esos nutrientes se implementó el método recomendado por el Consejo de Investigación Nacional (National Research Council) de los Estados Unidos (24). Aunque esta metodología es más simple y menos precisa desde el punto de vista estadístico, su empleo se justifica cuando los datos no cumplen los supuestos del método de Nusser y colaboradores.

Finalmente, se calculó para cada nutriente el cociente entre el MPLI y el respectivo requerimiento promedio estimado (EAR, sigla en inglés) $(25,26)$ en cada una de las mujeres, con el fin de obtener el porcentaje con ingestas inadecuadas. Las mujeres con cocientes MPLI/EAR $<1,0$ se clasificaron como el grupo con riesgo de deficiencia en la ingesta usual. Los EAR para cada nutriente se definen por grupo etario (cuadro 1). $\mathrm{Si}$ las mujeres hubieran pertenecido al mismo grupo de edad se podría haber 
CUADRO 1. Valores de referencia de los requerimientos promedio estimados para mujeres, según la edad

\begin{tabular}{lcc}
\hline & \multicolumn{2}{c}{ Edad (años) } \\
\cline { 2 - 3 } Nutriente/día & 14 a 18 & 19 a 49 \\
\hline Vitamina A $(\mu \mathrm{g})$ & 485 & 500 \\
Vitamina $_{6}(\mathrm{mg})$ & 1,0 & 1,1 \\
Vitamina $_{12}(\mu \mathrm{g})$ & 2,0 & 2,0 \\
Vitamina C $(\mathrm{mg})$ & 56,0 & 60,0 \\
Cinc $(\mathrm{mg})$ & 7,3 & 6,8 \\
Hierro $(\mathrm{mg})^{\mathrm{a}}$ & 14,2 & 14,6 \\
Cobre $(\mathrm{mg})$ & 0,685 & 0,700 \\
\hline
\end{tabular}

Fuente: Institute of Medicine. Otten J, Pitzi J, Meyers L. Dietary Reference Intakes. The Essential Guide to Nutrient Requirements. Washington, 2006.

a Valores utilizados en la Encuesta Nacional de la Situación Nutricional de Colombia, 2005.

estimado la prevalencia de consumos inadecuados simplemente calculando la proporción de mujeres con un consumo habitual inferior al EAR para el nutriente. En este trabajo las mujeres estudiadas pertenecían a distintos grupos de edad, y por eso fue necesario reescalar cada consumo habitual dividiéndolo por el EAR apropiado para cada mujer.

El proyecto contó con el aval del comité de bioética de la Sede de Investigación de la Universidad de Antioquia.

\section{Análisis estadístico}

Las distribuciones de la $\mathrm{Hb}$ y la ferritina presentaron una alta asimetría; por esto se describen en el cuadro 2 mediante sus respectivos percentiles.

Se compararon las proporciones de los tipos de anemia según las variables sociodemográficas. Para ello se utilizó la prueba F de Rao-Scott de segundo orden, que es una prueba corregida de la ji al cuadrado de Pearson. Se consideró significativa una probabilidad de $P<0,05$.

Se analizó la asociación entre la variable dependiente anemia (ferropénica y no ferropénica) y las variables independientes (nutrientes clasificados según el riesgo de deficiencia en la ingesta usual) mediante la razón de posibilidades (odds ratio, OR).

Los análisis se efectuaron con el software estadístico SPSS, versión 15.0 (SPSS Inc., Chicago, IL, EE.UU.), mediante la opción de análisis de muestras complejas; para el plan de muestreo se consideraron los departamentos de Colombia como variable de estratificación, y los municipios seleccionados en la encuesta como unidades primarias de muestreo.

Se estimó el efecto de diseño ("deff") como el cociente entre la varianza del estimador en estudio y la varianza del mismo estimador suponiendo que el muestreo hubiese sido aleatorio simple. Este estimador permite evaluar la eficiencia del diseño muestral complejo utilizado en la investigación, por lo que las estimaciones de medias y proporciones se presentan con sus respectivos efectos de diseño.

\section{RESULTADOS}

La muestra estuvo conformada por 595 mujeres con anemia, aparentemente sanas, sin proceso infeccioso activo; tenían diagnóstico de anemia no ferropénica $402(67,2 \%$; error estándar [EE] = $2,1 \%$; efecto de diseño [deff] $=1,2), y$ de anemia ferropénica 193 (32,8\%; EE = $2,1 \%$; deff $=1,2)$. La edad promedio de la muestra fue 25,6 años ( $\mathrm{EE}=0,4$ años; deff $=0,8) ; 29,4 \%(\mathrm{EE}=1,8 \%$; deff $=0,9)$ tenía entre 14 y 18 años; 39,0\% (EE = $2,4 \%$; deff $=1,4$ ) pertenecía al SISBEN 1; $21,0 \%(\mathrm{EE}=1,9 \%$; deff $=1,3)$ al SISBEN 3

CUADRO 2. Distribución en percentiles de los indicadores bioquímicos, según el tipo de anemia que padecen las mujeres en edad fértil en Colombia, 2005

\begin{tabular}{|c|c|c|c|c|c|c|c|c|c|}
\hline \multirow[b]{2}{*}{ Indicador bioquímico } & \multirow[b]{2}{*}{ Origen de la anemia } & \multirow[b]{2}{*}{ No. } & \multicolumn{7}{|c|}{ Percentil } \\
\hline & & & 5 & 10 & 25 & 50 & 75 & 90 & 95 \\
\hline \multirow[t]{2}{*}{ Hemoglobina (g/dL) } & Ferropénica & 195 & 7,9 & 8,9 & 9,9 & 10,6 & 11,3 & 11,7 & 11,9 \\
\hline & No ferropénica & 400 & 10,3 & 10,5 & 11,0 & 11,5 & 11,8 & 11,9 & 11,9 \\
\hline \multirow[t]{2}{*}{ Ferritina $(\mu \mathrm{g} / \mathrm{dL})$} & Ferropénica & 195 & 3,3 & 3,8 & 5,2 & 6,8 & 9,2 & 10,7 & 11,1 \\
\hline & No ferropénica & 400 & 13,5 & 15,0 & 19,5 & 29,0 & 44,4 & 62,0 & 84,1 \\
\hline
\end{tabular}

CUADRO 3. Comparación de los porcentajes del tipo de anemia, según las variables sociodemográficas de las mujeres en edad fértil con anemia en Colombia, 2005

\begin{tabular}{|c|c|c|c|c|c|c|}
\hline \multirow[b]{2}{*}{ Variable sociodemográfica } & \multirow[b]{2}{*}{ No. } & \multicolumn{2}{|c|}{ Tipo de anemia } & \multirow[b]{2}{*}{$\mathrm{EE}$} & \multirow[b]{2}{*}{ deff } & \multirow[b]{2}{*}{$P$} \\
\hline & & $\begin{array}{c}\text { No ferropénica } \\
\%\end{array}$ & $\begin{array}{c}\text { Ferropénica } \\
\%\end{array}$ & & & \\
\hline \multicolumn{7}{|l|}{ Edad (años) } \\
\hline 14 a 18 & 181 & 71,5 & 28,5 & 3,2 & 0,9 & 0,16 \\
\hline 19 a 49 & 414 & 65,4 & 34,6 & 2,7 & 1,3 & \\
\hline \multicolumn{7}{|l|}{ Área } \\
\hline Urbana & 485 & 66,9 & 33,1 & 2,4 & 1,3 & 0,79 \\
\hline Rural & 110 & 68,3 & 31,7 & 4,3 & 1,0 & \\
\hline \multicolumn{7}{|l|}{ SISBEN } \\
\hline 1 & 235 & 70,3 & 29,7 & 2,8 & 0,9 & 0,13 \\
\hline 2 & 239 & 68,3 & 31,7 & 2,9 & 0,9 & \\
\hline 3 a 6 & 121 & 59,4 & 40,6 & 5,6 & 1,6 & \\
\hline \multicolumn{7}{|l|}{ Región } \\
\hline Atlántica & 236 & 70,4 & 29,6 & 3,4 & 1,3 & 0,001 \\
\hline Oriental & 45 & 56,4 & 43,6 & 7,4 & 1,1 & \\
\hline Central & 80 & 65,8 & 34,2 & 5,6 & 1,3 & \\
\hline Pacífica & 56 & 47,9 & 52,1 & 7,9 & 1,5 & \\
\hline Bogotá & 27 & 56,0 & 44,0 & 8,6 & 1,2 & \\
\hline Orinoquia-Amazonia & 151 & 79,4 & 20,6 & 2,1 & 0,4 & \\
\hline
\end{tabular}

SISBEN, Sistema de Identificación de Potenciales Beneficiarios de Programas Sociales; EE, error estándar; deff, efecto de diseño; $P$ : probabilidad de la prueba de comparación de grupos en cada variable demográfica. 
CUADRO 4. Ingesta de nutrientes y frecuencia del riesgo de deficiencia en la ingesta usual, según el origen de la anemia en las mujeres en edad fértil en Colombia, 2005

\begin{tabular}{|c|c|c|c|c|c|c|c|c|}
\hline \multirow[b]{3}{*}{ Nutriente } & \multicolumn{4}{|c|}{ Cantidad ingerida } & \multicolumn{2}{|c|}{ Frecuencia del riesgo de deficiencia } & \multirow[b]{3}{*}{ OR } & \multirow[b]{3}{*}{$P$} \\
\hline & \multicolumn{2}{|c|}{$\begin{array}{c}\text { Anemia } \\
\text { ferropénica }\end{array}$} & \multicolumn{2}{|c|}{$\begin{array}{c}\text { Anemia } \\
\text { no ferropénica }\end{array}$} & \multirow{2}{*}{$\begin{array}{c}\begin{array}{c}\text { Anemia } \\
\text { ferropénica } \\
(n=402)\end{array} \\
\text { No. }(\%)\end{array}$} & \multirow{2}{*}{$\begin{array}{c}\text { Anemia } \\
\text { no ferropénica } \\
(n=193) \\
\text { No. }(\%)\end{array}$} & & \\
\hline & Media & EE & Media & $\mathrm{EE}$ & & & & \\
\hline Cobre (mg) & 1,00 & 0,04 & 0,96 & 0,04 & $152(37,5)$ & $62(32,1)$ & 1,27 & 0,13 \\
\hline Vitamina A (ER) & 809,8 & 83,1 & 835,7 & 77,7 & $231(57,5)$ & $96(49,8)$ & 1,36 & 0,053 \\
\hline Vitamina $\mathrm{B}_{6}(\mathrm{mg})$ & 0,56 & 0,14 & 0,53 & 0,13 & $362(90,1)$ & $170(88,1)$ & 1,22 & 0,38 \\
\hline Vitamina $B_{12}(\mu \mathrm{g})$ & 5,11 & 0,53 & 3,99 & 0,47 & $182(45,3)$ & $84(43,5)$ & 1,08 & 0,63 \\
\hline Hierro (mg) & 10,18 & 0,17 & 10,11 & 0,19 & $353(87,9)$ & $173(89,8)$ & 0,82 & 0,44 \\
\hline Vitamina C (mg) & 87,3 & 1,7 & 90,0 & 2,6 & $68(16,9)$ & $32(16,4)$ & 1,04 & 0,86 \\
\hline Cinc (mg) & 6,77 & 0,09 & 6,79 & 0,12 & $238(59,3)$ & $112(58,2)$ & 1,05 & 0,88 \\
\hline
\end{tabular}

$\mathrm{EE}$, error estándar; $\mathrm{OR}$, razón de posibilidades (odds ratio); $\mathrm{ER}$, equivalentes de retinol.

intervenciones nutricionales para prevenir o tratar la anemia están dirigidas a las mujeres gestantes (27). No existen programas para las mujeres en edad fértil. Esto hace a estas últimas más susceptibles a padecer dicha enfermedad durante el embarazo, tal como se corroboró en la ENSIN 2005, en la que se reportó que $44,7 \%$ de las mujeres embarazadas tenían anemia (2).

Colombia ha tenido la intención de realizar programas de intervención dirigidos a este grupo de población. Por esto, ha realizado algunos esfuerzos para identificar la prevalencia de anemia en las mujeres en edad fértil. Dentro de ellos se destaca la investigación realizada por Castro y colaboradores en 1995 (28). En esta investigación se definió como mujer en edad fértil a aquella que estaba embarazada y a la que tenía niños de entre 12 y 59 meses. Estos autores reportaron una prevalencia de anemia de $22,5 \%$ y de $19,6 \%$ de ferropenia. Posteriormente, la ENSIN 2005 halló que 32,8\% de las mujeres colombianas en edad fértil no embarazadas tuvieron anemia (2) y, aunque en ambos estudios se evaluó la ferritina sérica, en ninguno se identificó el origen de la anemia nutricional.

En nuestro estudio se observó que el porcentaje de mujeres con anemia no ferropénica fue el doble o más que el porcentaje de las que tenían anemia ferropénica en los dos grupos de edad, en las dos áreas, en los niveles de SISBEN 1 y 2 y en las regiones Atlántica, Central y Orinoquia-Amazonia.

Aunque la anemia ferropénica tuvo menor prevalencia, la distribución de los percentiles de $\mathrm{Hb}$ fue inferior a partir de la mediana comparada con aquella de las mujeres con anemia no ferropénica. La investigación indicó que en este grupo la anemia fue más severa. Este resultado cobra importancia, ya que otros estudios han observado que el diagnóstico de anemia y la severidad de la deficiencia de $\mathrm{Hb}$ durante el primer trimestre del embarazo se asocia con niños de bajo peso al nacer y con parto prematuro (29, 30). Por lo tanto, la anemia y la severidad de la deficiencia de $\mathrm{Hb}$ se convierten en un factor de riesgo para el tipo de mujeres incluidas en este estudio.

El análisis post hoc demostró que la prevalencia de anemia no ferropénica fue mayor en la región Orinoquia-Amazonia $(79,4 \%)$ que en la región Pacífica $(47,9 \%)$. Se desconocen las razones de este hecho y sería aventurado tratar de explicarlas, pues ambas regiones se caracterizan por una baja densidad de población y una gran biodiversidad. Tal vez, la única diferencia apreciable es que la primera región está habitada por población indígena, mestizos y blancos, y en la segunda predomina la población de ascendencia africana.

Establecer relaciones entre los indicadores bioquímicos y las deficiencias nutricionales es una tarea difícil ya que, normalmente, no se obtienen asociaciones estadísticas fuertes. Esto, se debe a que no es posible controlar todas las variables en la medición de la ingesta usual y a que el organismo humano posee múltiples mecanismos de adaptación fisiológica para garantizar la homeostasis nutricional y su funcionamiento. Esto conduce a que el período de latencia entre la carencia nutricional y su manifestación pueda ser de semanas a años. Las razones expuestas han llevado a los expertos a considerar la evaluación de la dieta humana como un proceso com- plejo, difícil, impreciso y problemático. No obstante, destacan su importancia en el diseño de intervenciones dirigidas a prevenir o a reducir las consecuencias negativas de las deficiencias nutricionales (12, 31-33). Posiblemente, las razones expuestas sean la causa por la cual no se encontró una asociación entre la ingesta de nutrientes y el tipo de anemia.

En la búsqueda bibliográfica no se hallaron estudios que coincidieran con los criterios establecidos en esta investigación, lo que dificultó la comparación. Sin embargo, es importante destacar los siguientes resultados: Mousa y colaboradores (12) encontraron que las adolescentes de Kuwait con anemia tuvieron mayor carencia en la ingesta dietética que las jóvenes sin este diagnóstico; Ronnenberg y colaboradores (34) evaluaron los indicadores bioquímicos de mujeres en edad fértil en China, trabajadoras de una empresa textil. Encontraron una prevalencia de anemia de $80 \%$, pero sólo el $18 \%$ de las personas afectadas presentaron depleción de los depósitos de hierro. No obstante, $54 \%$ tuvieron deficiencia bioquímica de una o más vitaminas del complejo B; estos autores aclaran que no evaluaron la presencia de infecciones y que, por tanto, existe la posibilidad de falsos positivos de ferritina normal. Sin embargo, destacan que pudieron existir otras deficiencias nutricionales que afectaron la síntesis de la $\mathrm{Hb}$. Ahmed y colaboradores $(19,35)$ también encontraron que coexistían deficiencias bioquímicas de hierro, vitamina $\mathrm{A}$, riboflavina $\mathrm{y}$ ácido fólico en mujeres adolescentes con anemia en Bangladesh, y que tanto la $\mathrm{Hb}$ como los otros indicadores bioquímicos mejoraron después de la administración de un suplemento multivitamínico. Or- 
tega y colaboradores (36) evaluaron adolescentes en Venezuela y encontraron que solo 26,9\% de las participantes tenían anemia ferropénica. En otra investigación, llevada a cabo en mujeres gestantes de Antioquia, Colombia, se comprobaron deficiencias en la ingesta de nutrientes hematopoyéticos y en los indicadores bioquímicos evaluados durante el primer trimestre de embarazo. Estos valores se corrigieron a partir del segundo trimestre de gestación, después de que las mujeres participaron en un programa de intervención nutricional $(37,38)$.

Los resultados de esas investigaciones concuerdan con los hallazgos de nuestro estudio, en el sentido que las participantes tuvieron deficiencia de varios nutrientes necesarios para la síntesis de la $\mathrm{Hb}$ y en que hubo un alto porcentaje de mujeres con anemia no ferropénica.

Las limitaciones de este estudio fueron la imposibilidad de analizar el riesgo de deficiencia de ácido fólico en la ingesta usual debido a que las unidades de medida (mg) de las tablas de composición colombianas no coinciden con las establecidas en la recomendaciones nutri- cionales (microgramos de equivalentes de folato dietario) y que en el análisis no se incluyó el diagnóstico de parásitos helmínticos y de paludismo, el número de hijos, el espacio intergenésico, las características de la menstruación ni el método de planificación familiar empleado por las mujeres. Sin embargo, los resultados permiten concluir que la mayoría de las mujeres en edad fértil de Colombia padecen anemia ocasionada por causas diferentes a la deficiencia de hierro, excepto en la región Pacífica en la cual se observó el mayor porcentaje de mujeres con anemia ferropénica. Además, se encontró una alta prevalencia de deficiencia de nutrientes hematopoyéticos en la ingesta usual en todas las mujeres del estudio. No se observó asociación estadísticamente significativa entre las deficiencias en el consumo de estos nutrientes y el origen de la anemia nutricional.

Los hallazgos permiten hacer dos recomendaciones. En primer lugar, es necesario planear e implementar programas orientados a mejorar el aporte de nutrientes hematopoyéticos en las mujeres en edad fértil en Colombia. De este modo, se promueve la formación de reservas adecuadas y, por ende, aumenta la disponibilidad para responder a las altas demandas del embarazo. Esto se traduciría en efectos positivos para las mujeres, los niños y el país. En segundo lugar, es necesario desarrollar investigaciones orientadas a buscar otras causas de la anemia, diferentes a la deficiencia de hierro. Esto sería muy positivo para diseñar estrategias más contextualizadas de prevención de la anemia nutricional, que logren un impacto más efectivo.

Agradecimientos. Al Instituto Colombiano de Bienestar Familiar, por facilitar las bases de datos de la ENSIN 2005. A la Escuela de Nutrición y Dietética y a la Universidad de Antioquia, por el apoyo mediante la estrategia de sostenibilidad 2011-2012.

Conflicto de intereses. Luz Mariela Manjarrés es asesora en aspectos de nutrición del Laboratorio Profesional Farmacéutico LAPROFF S.A., Sabaneta, Colombia.

\section{REFERENCIAS}

1. MacLean E, Egli I, Benoist B, Wojdyla D, Cogswell M. Worldwide prevalence of anemia in pre-school age children, pregnant women and non-pregnant women of reproductive age. En: Kraemer K, Zimmermann M, editors. Nutritional Anemia. Basel: Sight and life Press; 2007. Pp. 1-13.

2. Instituto Colombiano de Bienestar Familiar, Profamilia, Instituto Nacional de Salud, Universidad de Antioquia, OPS. Valoración del Estado nutricional por indicadores bioquímicos. En: Borda C, editor. Encuesta nacional de la situación nutricional en Colombia, 2005. Bogotá: Panamericana Formas e Impresos S.A.; 2006. Pp. 123-63.

3. Gleason G, Scrimshaw N. An overview of the functional significance of iron deficiency. En: Kraemer K, Zimmermann M, editors. Nutritional Anemia. Basel: Sight and life Press; 2007. Pp. 46-57.

4. Bocio J, Páez C, Zubillaga M. Causas y consecuencias de la deficiencia de hierro sobre la salud humana. ALAN. 2004;54(2):165-73.

5. World Health Organization, Centers for Disease Control and Prevention. Atlanta. Worldwide prevalence of anaemia 1993-2005. WHO Global database on anaemia Switzerland: World Health Organization, 2008.

6. Olivares M, Walter T. Causas y consecuencias de la deficiencia de hierro. Revista de Nutrición. 2004;17(1):5-14.

7. Bhutta Z, Ahmed T, Black R, Couusens S. What works? Interventions for maternal and child undernutrition and survival. Lancet. 2008;371:417-39.

8. Dallman R, Yip R, Johnson C. Prevalence and causes of anemia in the Unites States, 1976 to 1980. Am J Clin Nutr. 1984;39:437-45.

9. Pizarro F, Calvo E. El significado, a mediano y largo plazo, de la deficiencia de hierro y zinc durante los primeros dos años de vida, para asegurar un buen crecimiento temprano. En: Uauy R, Carmuega E, editors. Impacto del crecimiento y desarrollo sobre la salud y bienestar de la población. Perspectivas y reflexiones del cono sur. Buenos Aires: Instituto Danone Cono Sur; 2009. Pp. 49-64.

10. Center of Disease Control and Prevention. Assessing the iron Status of The Population Report. A joint technical consultation on the assessment of iron status at the population level. Switzerland, 2004.

11. Kraemer K, Zimmermann M. Nutritional Anemia. Basel: Sight and life; 2007.

12. Al Mousa Z, Prakash P, Jackson RT, Al Raqua $M$. A comparison of selected nutrient intakes in anemic and nonanemic adolescent girls in Kuwait. Nutrition Research. 2003;23(4):425-33. doi: 10.1016/S0271-5317(02) 00543-2.

13. Shane B. Folic Acid, Vitamin B12 and vitamin B6. En: Stipanuk M, editor. Biochemical, Physiological, Molecular Aspects of Human Nutrition. 2 ed. New York: Saunders Elsevier; 2006. Pp. 793-59.
14. Vinodkumar M, Rajagopalan S. Efficacy of fortification of school meals with ferrous glycine phosphate and riboflavin against anemia and angular stomatitis in schoolchildren. Food Nutr Bull. 2009;30(3):260-4.

15. FAO/WHO. Folate and folic acid. Human Vitamin and Mineral Requirements. Rome: Food and Nutrition Division; 2001. Pp. 53-61.

16. Scott J. Nutritional anemia: B-Vitamins. En: Kraemer K, Zimmermann M, editors. Nutritional Anemia. Basel: Sight and life Press; 2007. Pp. 112-32.

17. Institute of Medicine. Folate. En: Otten J, Hellwig J, Meyeres L, editors. Dietary Reference Intakes The Essential guide to Nutrient Requirements. Washington D.C: The National Academies Press; 2006. Pp. 244-53.

18. Hess S, Lönnerdal B, Hotz C, Rivera J, Brown $\mathrm{K}$. Recent advances in knowledge of zinc nutrition and human health. Food Nutr Bull. 2009;30(S)(1):5S-11S.

19. Ahmed F, Khan M, Akhtaruzzaman M, Karim $\mathrm{R}$, Williams $\mathrm{G}$, Torlesse H, et al. Long-term intermittent multiple micronutrient supplementation enhances hemoglobin and micronutrient status more than iron + folic acid supplementation in Bangladeshi rural adolescent girls with nutritional anemia. J Nutr. 2010;140(10):1879-86.

20. Instituto Colombiano de Bienestar Familiar, Profamilia, Instituto Nacional de Salud, Universidad de Antioquia, OPS. Metodología. En: Borda C, editor. Encuesta nacional de la 
situación nutricional en Colombia, 2005. Bogotá: Panamericana Formas e Impresos; 2006. Pp. 27-49.

21. Manjarrés L. Métodos para precisar la recolección de la ingesta en estudios poblacionales. Perspectivas en Nutrición Humana. 2007;9(2):155-63.

22. Manjarrés L, Correa J. Programa de Evaluación de Ingesta Dietética. Escuela de Nutrición y Dietética. $3^{\text {a }}$ ed. Medellín: Escuela de Nutrición y Dietética.Universidad de Antioquia; 2006.

23. Nusser SM, Carriquiry AL, Dodd KW, Fuller WA. A Semiparametric Transformation Approach to Estimating Usual Daily Intake Distributions. J Am Stat Assoc. 1996;91(436): 1440-9.

24. National Research Council. Nutrient Adequacy. Washington, DC: National Academy Press; 1986.

25. Institute of Medicine. Dietary Reference Intakes. The Essential Guide to Nutrient Requirements. Washington, D.C: The National Academy Press; 2006.

26. Instituto Colombiano de Bienestar Familiar, Profamilia, Instituto Nacional de Salud, Universidad de Antioquia, OPS. Ingesta Dietética. En: Borda C, editor. Encuesta nacional de la situación nutricional en Colombia, 2005. Bogotá: Panamericana Formas e Impresos S.A.; 2006. Pp. 229-61.

27. Shrimpton R, Huffman SL, Zehner ER, Darnton-Hill I. Multiple micronutrient supplementation during pregnancy in developingcountry settings: Policy and program impli- cations of the results of a meta analysis. Food Nutr Bull. 2009;30(4 Suppl):S 556-73.

28. Castro de Navarro L, Nicholls S. Deficiencia de hierro, vitamina A y parasitismo intestinal en la población infantil y la anemia nutricional en mujeres en edad fértil. Colombia 1995-1996. Santa Fé de Bogotá, D.C: Instituto Nacional de Salud; 1998.

29. Zhou L, Yang W, Hua J, Deng C, Tao X, Stolzfus R. Relation of hemoglobin measured at different times in pregnancy to preterm birth and low birth weight in Shanghai,China. Am J Epidemiol. 1998;148:998-1006.

30. Scanlon K, Yip R, Shieve L, Cogswell M. High and low hemoglobin levels during pregnancy: differential risks for preterm birth and small for gestational age. Obstet Gynecol. 2000;96(5 Pt 1):741-8.

31. Ajit Chiplonkar S, Vilas Agte V, Shashikant Mengale S. Relative importance of micronutrient deficiencies in iron deficiency anemia. Nutrition Research. 2003;23(10):1355-67.doi: 10.1016/S0271-5317(03)00151-9.

32. Willet W. Overview of nutritional epidemiology. En: Willet W, editor. Nutritional Epidemiology. 2 ed. New York: Oxford University Press; 1998. Pp. 3-17.

33. Yamamoto M. Analytic Nutrition Epidemiology. En: Monsen E, Van Horn E, editors. Research Successful Approaches. 3 ed. Chicago: American Dietetic Association; 2007. Pp. 81-100.

34. Ronnenberg A, Goldman M, Aitken I, Xu X. Anemia and Deficiencies of Folate and Vitamin B-6 Are Common and Vary with Season in Chinese Women of Childbearing Age. J Nutr. 2000;130:2703-10.

35. Ahmed F, Khan M, Banu C, Qazi M, Akhtaruzzaman $\mathrm{M}$. The coexistence of other micronutrient deficiencies in anaemic adolescent schoolgirls in rural Bangladesh. Eur J Clin Nutr. 2008;62:365-72.

36. Ortega P, Leal J, Amaya D, Chávez C. Evaluación nutricional, deficiencia de micronutrientes y anemia en adolescentes femeninas de una zona urbana y una rural del estado de Zulia, Venezuela. Invest Clin. 2010;51:37-52.

37. Manjarrés L, Díaz A. Cambios en el aporte de energía y nutrientes en un grupo de mujeres gestantes participantes del programa MANA para la vida. Gobernación de Antioquia. En: Restrepo S, editor. Alimentación y nutrición de la mujer gestante. Diagnóstico y lineamientos para la acción. Medellín: Divergráficas Ltda; 2007. Pp. 137-81.

38. Parra B. Evaluación bioquímica del estado nutricional del hierro y folato en un grupo de mujeres gestantes participantes en el programa MANA por la vida. En: Restrepo S, editor. Alimentación y nutrición de la mujer gestante. Diagnóstico y lineamientos para la acción. Medellín: Gobernación de Antioquia; 2007. Pp. 217-43.

Manuscrito recibido el 6 de octubre de 2010. Aceptado para publicación, tras revisión, el 2 de junio de 2011.

ABSTRACT Objectives. Compare the nutritional origin of anemia by sociodemographic variables and analyze its association with deficient hematopoietic nutrient intake.

Methods. The database of Colombia's 2005 National Survey of Nutritional Status

Association between hematopoietic nutrient intake and the origin of nutritional anemia in women of childbearing age in Colombia was used. The data were obtained through complex representative sampling of the population and processed using SPSS v.15. Anemic women of childbearing age were selected and divided into two groups according to serum ferritin levels. Their customary hematopoietic nutrient intake and risk of deficiency were determined. The proportions of anemia types were compared by sociodemographic variables using the $F$-distribution, the Rao-Scott second order correction $(P<0.05)$. The association between the origin of the anemia and classification of the nutrient was analyzed using the odds ratio (OR).

Results. Sample: 595 women. Non-hypoferric anemia (67.2\%) predominated, with no statistical difference by sociodemographic variable, except in the Pacific region (hypoferric anemia, 52.1\%). The prevalence of deficiency in the customary intake of hematopoietic nutrients was high. There was no significant association between the deficit in consumption and the origin of the anemia.

Conclusions. Non-hypoferric anemia was most common, with no difference by sociodemographic indicators except in the Pacific region. All the women were at high risk of deficiency in their customary hematopoietic nutrient intake, but a statistically significant association between the deficiency and the origin of the nutritional anemia was not observed. Programs to improve nutrient intake and a continued search for causes of nutritional anemia other than iron deficiency are justified.

Key words Anemia; nutritional anemias; ferritins; avitaminosis; iron deficiency; vitamin B deficiency; women's health; Colombia. 